\section{CT: Krebs durch niedrige Strahlendosis?}

\section{Erkenntnisse an Überlebenden von Atombombenexplosionen lassen vermuten, dass medizinisch genutzte Strahlendosen das Krebsrisiko erhöhen. Britische Forscher haben versucht, dies bei Kindern und Jugendlichen zu klären.}

B ereits vor mehr als einer Dekade lieBen Modellrechnungen aufgrund der Erkenntnisse an Überlebenden der Atombombenexplosionen in Japan vermuten, dass auch in der Medizin genutzte niedrige Strahlendosen das Krebsrisiko erhöhen. Jetzt liefert eine britische retrospektive Kohortenstudie Daten von fast 180.000 Patienten, die als nicht an Krebs erkrankte Kinder zwischen 1985 und 2002 eine Computertomografie (CT)-Untersuchung bekamen.

Bei insgesamt 74 von 178.604 Patienten wurde nachfolgend eine Leukämie diagnostiziert, bei 135 von 176.587 ein Hirntumor. Die britischen Wissenschaftler um den Radiologen Mark Pearce stellten bei der Auswertung der Daten fest, dass die Leukämie-Inzidenz bei Kindern deutlich erhöht war, wenn ihr Knochenmark einer kumulativen Dosis von mindestens 30 mGy ausgesetzt war.
Der Anstieg der Hirntumor-Inzidenz war signifikant bei einer kumulativen Dosis von mindestens 50 mGy. Die Forscher gehen davon aus, dass bei Kindern, die jünger als 15 Jahre sind, durch zwei bis drei CT-Aufnahmen des Kopfes mit solchen Strahlendosen das Risiko für Hirntumoren sowie durch fünf bis zehn CT-Aufnahmen des Kopfes das Leukämierisiko verdreifacht wird. Vergleichsgruppe waren Patienten mit einer Dosis unter $5 \mathrm{mGy}$.

Nach Angaben der Wissenschaftler geht aus ihren Berechnungen hervor, dass von 10.000 Menschen im Alter bis zu 20 Jahren, die durch eine CT-Untersuchung eine Strahlendosis von $10 \mathrm{mGy}$ abbekommen, ein Patient zusätzlich an einer Leukämie erkrankt oder von 30.000 Menschen zusätzlich ein Patient an einem Tumor des zentralen Nervensystems.
Anders ausgedrückt: Eine CT-Aufnahme des Kopfes bei Kindern bis zu zehn Jahren führt pro 10.000 Patienten innerhalb einer Dekade nach der Untersuchung zu einer zusätzlichen Leukämie oder einem zusätzlichen Hirntumor.

Die Wissenschaftler legen Wert auf die Feststellung, dass CT-Untersuchungen weiterhin nur dann gemacht werden sollten, wenn sie klinisch erforderlich und entsprechende Untersuchungsergebnisse nicht von anderen bildgebenden Verfahren zu erwarten sind. Zudem sollte die Entwicklung von CT-Geräten mit noch niedrigeren Strahlenbelastungen vorangetrieben werden.

Fazit: Kinder und Jugendliche, die sich mehrmals einer CT-Untersuchung unterziehen müssen, haben trotz der geringen Strahlendosis ein erhöhtes Risiko für Leukämie oder Hirntumoren. Allerdings ist dieses Risiko nur leicht erhöht. Die Entscheidung für CT-Untersuchungen sollte dennoch mit Bedacht gefällt werden.

Peter Leiner

Pearce MS et al. Radiation exposure from CT scans in childhood and subsequent risk of leukaemia and brain tumours: A retrospective cohort study. Lancet. 2012;380(9840):499-505.

\title{
DSRCT - seltene Neoplasie im Fokus
}

\section{Der desmoplastische klein- und rundzellige Tumor ist ein sehr seltenes und aggressives Malignom, für das es keine einheitlichen Therapieempfehlungen gibt. Eine retrospektive Studie bringt mehr Klarheit über diese Tumorentität.}

$\mathrm{D}$ ie Tumoren sind am häufigsten im Abdominalraum. Es kommt schnell zur peritonealen Streuung. Der „desmoplastic small round cell tumor" (DSRCT) zeigt eine charakteristische genetische Veränderung: Das EWS-WT1-Fusionstranskript kann durch Polymerase-Kettenreaktion nachgewiesen werden.

Eine französische Autorengruppe arbeitete eine Serie von 38 Fällen mit diesen Tumoren auf. Sie beschränkte sich auf Patienten im Alter unter 30 Jahren mit kompletten klinischen Daten. $26 \mathrm{~Pa}$ tienten zeigten gastrointestinale Symptome (meist Schmerzen), das mediane Tumorvolumen betrug $90 \mathrm{~cm}^{3}$. Der Primärtumor lag bei $87 \%$ der Patienten ab- dominal und/oder im Becken - in Einzelfällen waren Knochen, Lunge, Mediastinum oder Gehirn betroffen. Nur bei neuen Patienten $(24 \%)$ war der Tumor lokal begrenzt. Die häufigsten Fernmetastasen zeigten sich mit $42 \%$ in der Leber. In jeweils über $10 \%$ wurden mediastinale, ossäre, pulmonale und in der Pleura gelegene Metastasen gefunden.

30 Patienten erhielten eine neoadjuvante bzw. initiale Chemotherapie, bei 14 wurde im Anschluss eine Tumorresektion durchgeführt. Bei sechs erfolgte eine Operation erst später nach einer Tumorprogression. Alle Patienten mit primärer Resektion erhielten im Anschluss eine adjuvante Chemotherapie. Die ein- gesetzten Chemotherapien waren sehr variabel, am häufigsten kamen Alkylanzien und Anthrazykline zum Einsatz.

Die 2-Jahres-Raten für das Gesamtüberleben lagen bei $50 \%$, für das ereignisfreie Überleben bei $14,5 \%$. In der univariaten Analyse war die komplette Tumorresektion der einzige Faktor, der das Gesamtüberleben signifikant verlängerte $(\mathrm{p}=0,041)$, beim Fehlen von Fernmetastasen zum Diagnosezeitpunkt zeigte sich mit $\mathrm{p}=0,073$ ein Trend. Weder Radio- noch Hochdosis-Chemotherapie verbesserten das Überleben signifikant.

Fazit: Der DSRCT ist ein Tumor mit äuBerst schlechter Prognose. Patienten nach kompletter Tumorresektion haben noch die besten Langzeit-Überlebenschancen.

Andreas Fischer

Philippe-Chomette P et al. Desmoplastic small round cell tumors with EWS-WT1 fusion transcript in children and young adults. Pediatr Blood Cancer. 2012;58(6):891-7. 